\title{
Existence and Multiplicity of Positive Solutions for a System of Fourth-Order Boundary Value Problems
}

\author{
Shoucheng Yu and Zhilin Yang \\ Department of Mathematics, Qingdao Technological University, No. 11 Fushun Road, Qingdao, Shandong 266033, China \\ Correspondence should be addressed to Zhilin Yang; zhilinyang@sina.com
}

Received 26 February 2014; Accepted 15 April 2014; Published 3 June 2014

Academic Editor: Salvatore A. Marano

Copyright (C) 2014 S. Yu and Z. Yang. This is an open access article distributed under the Creative Commons Attribution License, which permits unrestricted use, distribution, and reproduction in any medium, provided the original work is properly cited.

We study the existence and multiplicity of positive solutions for the system of fourth-order boundary value problems $x^{(4)}=$ $f\left(t, x, x^{\prime},-x^{\prime \prime},-x^{\prime \prime \prime}, y, y^{\prime},-y^{\prime \prime},-y^{\prime \prime \prime}\right), y^{(4)}=g\left(t, x, x^{\prime},-x^{\prime \prime},-x^{\prime \prime \prime}, y, y^{\prime},-y^{\prime \prime},-y^{\prime \prime \prime}\right), x(0)=x^{\prime}(1)=x^{\prime \prime}(0)=x^{\prime \prime \prime}(1)=0$, and $y(0)=y^{\prime}(1)=y^{\prime \prime}(0)=y^{\prime \prime \prime}(1)=0$, where $f, g \in C\left([0,1] \times \mathbb{R}_{+}^{8}, \mathbb{R}_{+}\right)\left(\mathbb{R}_{+}:=[0, \infty)\right)$. We use fixed point index theory to establish our main results based on a priori estimates achieved by utilizing some integral identities and inequalities and $\mathbb{R}_{+}^{2}$-monotone matrices.

\section{Introduction}

In this paper we study the existence and multiplicity of positive solutions for the system of fourth-order boundary value problems:

$$
\begin{gathered}
x^{(4)}=f\left(t, x, x^{\prime},-x^{\prime \prime},-x^{\prime \prime \prime}, y, y^{\prime},-y^{\prime \prime},-y^{\prime \prime \prime}\right), \\
y^{(4)}=g\left(t, x, x^{\prime},-x^{\prime \prime},-x^{\prime \prime \prime}, y, y^{\prime},-y^{\prime \prime},-y^{\prime \prime \prime}\right), \\
x(0)=x^{\prime}(1)=x^{\prime \prime}(0)=x^{\prime \prime \prime}(1)=0, \\
y(0)=y^{\prime}(1)=y^{\prime \prime}(0)=y^{\prime \prime \prime}(1)=0,
\end{gathered}
$$

where $f, g \in C\left([0,1] \times \mathbb{R}_{+}^{8}, \mathbb{R}_{+}\right)\left(\mathbb{R}_{+}:=[0, \infty)\right)$. It is well known that the deflection of elastic beams can be described by some fourth-order boundary value problems; for example, see $[1,2]$. Consequently, fourth-order boundary value problems play a very important role in both theory and applications. Boundary value problems for systems of nonlinear ordinary differential equations have been also studied by several authors; for example, see [3-11] and the references therein. In [3], Li et al. use fixed point theorems on cones to establish the existence of positive solutions for a system of third-order boundary value problems:

$$
\begin{gathered}
-u^{\prime \prime \prime}=a(t) f(t, v), \\
-v^{\prime \prime \prime}=b(t) h(t, u), \\
u(0)=u^{\prime}(0)=0, \quad u^{\prime}(1)=\alpha u^{\prime}(\eta), \\
v(0)=v^{\prime}(0)=0, \quad v^{\prime}(1)=\alpha v^{\prime}(\eta) .
\end{gathered}
$$

In [4], Lü et al. study the existence of positive solutions for a system of boundary value problems:

$$
\begin{gathered}
u^{(4)}=f(t, v), \quad-v^{\prime \prime}=g(t, u), \quad t \in(0,1), \\
u(0)=u(1)=u^{\prime \prime}(0)=u^{\prime \prime}(1)=v(0)=v(1)=0,
\end{gathered}
$$

where $f, g \in C\left([0,1] \times \mathbb{R}_{+}, \mathbb{R}_{+}\right)$. In [11], the authors investigate the existence and multiplicity of positive solutions for the system

$$
\begin{gathered}
(-1)^{m} w^{(2 m)}=f\left(t, w, w^{\prime},-w^{\prime \prime \prime}, \ldots,(-1)^{m-1} w^{(2 m-1)},\right. \\
\left.z, z^{\prime},-z^{\prime \prime \prime}, \ldots,(-1)^{n-1} z^{(2 n-1)}\right),
\end{gathered}
$$




$$
\begin{array}{r}
(-1)^{n} z^{(2 n)}=g\left(t, w, w^{\prime},-w^{\prime \prime \prime}, \ldots,(-1)^{m-1} w^{(2 m-1)},\right. \\
\left.z, z^{\prime},-z^{\prime \prime \prime}, \ldots,(-1)^{n-1} z^{(2 n-1)}\right), \\
w^{(2 i)}(0)=w^{(2 i+1)}(1)=0 \quad(i=0,1, \ldots, m-1), \\
z^{(2 j)}(0)=z^{(2 j+1)}(1)=0 \quad(j=0,1, \ldots, n-1) .
\end{array}
$$

The hypotheses imposed on the nonlinearities $f$ and $g$ are formulated in terms of two linear functions $h_{1}(x)$ and $h_{2}(y)$. The main results in [11] are established by using fixed point index theory based on a priori estimates of positive solutions achieved by utilizing new integral inequalities and nonnegative matrices.

This paper is organized as follows. In Section 2, we use the method of order reduction to transform (1) into a system of boundary value problems for second-order integrodifferential equations. Also, in this section, we develop some basic integral identities and inequalities that are useful in deriving a priori estimates in Section 3. Our main results, namely, Theorems 11-14, are stated and proved in Section 3. Finally, three examples that illustrate our main results are presented in Section 4.

\section{Preliminaries}

Let $E:=C^{1}([0,1], \mathbb{R}),\|u\|:=\max \left\{\|u\|_{0},\left\|u^{\prime}\right\|_{0}\right\}\left(\|u\|_{0}:=\right.$ $\max \{|u(t)|: t \in[0,1]\}), P:=\left\{u \in E: u(t) \geqslant 0, u^{\prime}(t) \geqslant\right.$ $0, \forall t \in[0,1]\}$. Then $(E,\|\cdot\|)$ is a real Banach space and $P$ is a solid cone in $E$. Let

$$
k(t, s):=\min \{t, s\}, \quad 0 \leqslant t, s \leqslant 1 .
$$

Define the linear integral operator $B$ by

$$
(B \omega)(t):=\int_{0}^{1} k(t, s) \omega(s) \mathrm{d} s
$$

It is well known that $B: E \rightarrow E$ is a completely continuous, positive, linear operator and

$$
(B \psi)(s)=\int_{0}^{1} k(t, s) \psi(t) \mathrm{d} t=r(B) \psi(s),
$$

where $\psi(t):=\sin (\pi / 2) t$. Substituting $u(t):=-x^{\prime \prime}(t)$, $v(t):=-y^{\prime \prime}(t)$ into (1), we transform (1) into the following system of seconed-order boundary value problems for the integrodifferential equations:

$$
\begin{aligned}
& -u^{\prime \prime}=f\left(t, B u,(B u)^{\prime}, u, u^{\prime}, B v,(B v)^{\prime}, v, v^{\prime}\right), \\
& -v^{\prime \prime}=g\left(t, B u,(B u)^{\prime}, u, u^{\prime}, B v,(B v)^{\prime}, v, v^{\prime}\right), \\
& u(0)=u^{\prime}(1)=0, \quad v(0)=v^{\prime}(1)=0,
\end{aligned}
$$

which is equivalent to the system of nonlinear integral equations:

$$
\begin{array}{r}
u(t)=\int_{0}^{1} k(t, s) f\left(s,(B u)(s),(B u)^{\prime}(s), u(s), u^{\prime}(s),\right. \\
\left.(B v)(s),(B v)^{\prime}(s), v(s), v^{\prime}(s)\right) \mathrm{d} s, \\
v(t)=\int_{0}^{1} k(t, s) g\left(s,(B u)(s),(B u)^{\prime}(s), u(s), u^{\prime}(s),\right. \\
\left.(B v)(s),(B v)^{\prime}(s), v(s), v^{\prime}(s)\right) \mathrm{d} s .
\end{array}
$$

Define the operators $A_{i}(i=1,2): P^{2} \rightarrow P$ and $A: P^{2} \rightarrow$ $P^{2}$ by

$$
\begin{array}{r}
A_{1}(u, v)(t)=\int_{0}^{1} k(t, s) f\left(s,(B u)(s),(B u)^{\prime}(s), u(s), u^{\prime}(s),\right. \\
\left.(B v)(s),(B v)^{\prime}(s), v(s), v^{\prime}(s)\right) \mathrm{d} s, \\
A_{2}(u, v)(t)=\int_{0}^{1} k(t, s) g\left(s,(B u)(s),(B u)^{\prime}(s), u(s), u^{\prime}(s),\right. \\
\left.(B v)(s),(B v)^{\prime}(s), v(s), v^{\prime}(s)\right) \mathrm{d} s,
\end{array}
$$$$
A(u, v)(t)=\left(A_{1}(u, v)(t), A_{2}(u, v)(t)\right) .
$$

If $f, g \in C\left([0,1] \times \mathbb{R}_{+}^{8}, \mathbb{R}_{+}\right)$, then $A_{i}$ and $A$ are completely continuous operators. Clearly, the existence of positive solutions for (1) is equivalent to that of positive fixed points of $A: P^{2} \rightarrow P^{2}$. Lemmas $1-4$ below are cited from [12].

Lemma 1. If $\omega \in P \cap C^{2}[0,1], \omega(0)=\omega^{\prime}(1)=0$, then

$$
\int_{0}^{1}\left(-\omega^{\prime \prime}(t)\right) t e^{t} \mathrm{~d} t=\int_{0}^{1}\left(\omega(t)+2 \omega^{\prime}(t)\right) t e^{t} \mathrm{~d} t .
$$

Lemma 2. If $\omega \in P$, then

$$
\int_{0}^{1}\left((B \omega)(t)+2(B \omega)^{\prime}(t)\right) t e^{t} \mathrm{~d} t=\int_{0}^{1} \omega(t) t e^{t} \mathrm{~d} t
$$

Lemma 3. If $\omega \in P, \omega(0)=0$, and $\omega^{\prime}$ is decreasing on $[0,1]$, then

$$
\int_{0}^{1} \omega(t) t e^{t} \mathrm{~d} t \geqslant(e-2) \int_{0}^{1} \omega^{\prime}(t) t e^{t} \mathrm{~d} t
$$

Lemma 4. If $\omega \in P, \omega(0)=0$, then

$$
\omega(1) \leqslant \int_{0}^{1}\left(\omega(t)+2 \omega^{\prime}(t)\right) t e^{t} \mathrm{~d} t .
$$

Lemma 5. Suppose $\psi \in C\left([0,1], \mathbb{R}_{+}\right)$is not identically vanishing on $[0,1]$, and $\omega \in C\left([0,1], \mathbb{R}_{+}\right)$is a concave function. Let $\varrho(\psi):=\int_{0}^{1} t \psi(t) \mathrm{d} t>0$. Then

$$
\int_{0}^{1} \psi(t) \omega(t) \mathrm{d} t \geqslant \omega(1) \varrho(\psi) .
$$


Proof. By the concavity of $v$ and the nonnegativity of $\psi$, we have

$$
\begin{aligned}
\int_{0}^{1} \psi(t) \omega(t) \mathrm{d} t & =\int_{0}^{1} \psi(t) \omega(t \cdot 1+(1-t) \cdot 0) \mathrm{d} t \\
& \geqslant \omega(1) \int_{0}^{1} t \psi(t) \mathrm{d} t=\omega(1) \varrho(\psi) .
\end{aligned}
$$

This completes the proof.

Lemma 6 (see [13]). Let $E$ be a real Banach space and $P$ a cone in $E$. Suppose that $\Omega \subset E$ is a bounded open set and that $T: \bar{\Omega} \cap P \rightarrow P$ is a completely continuous operator. If there exists $\omega_{0} \in P \backslash\{0\}$ such that

$$
\omega-T \omega \neq \lambda \omega_{0}, \quad \forall \lambda \geqslant 0, \omega \in \partial \Omega \cap P,
$$

then $i(T, \Omega \cap P, P)=0$, where $i$ indicates the fixed point index on $P$.

Lemma 7 (see [13]). Let $E$ be a real Banach space and $P$ a cone in $E$. Suppose that $\Omega \subset E$ is a bounded open set with $0 \in \Omega$ and that $T: \bar{\Omega} \cap P \rightarrow P$ is a completely continuous operator. If

$$
\omega-\lambda T \omega \neq 0, \quad \forall \lambda \in[0,1], \omega \in \partial \Omega \cap P,
$$

then $i(T, \Omega \cap P, P)=1$.

Lemma 8 (see [14, Lemma 2.4]). If $p$ is concave on $[d, \infty$ ), with $\lim _{y \rightarrow \infty} p(y) / y \geq 0$, then $p$ is increasing on $[d, \infty)$ and

$$
p(y+z-d) \leqslant p(y)+p(z)-p(d)
$$

for all $y, z \in[d, \infty)$.

\section{Main Results}

Let $x:=\left(x_{1}, x_{2}, x_{3}, x_{4}\right) \in \mathbb{R}_{+}^{4}, y:=\left(y_{1}, y_{2}, y_{3}, y_{4}\right) \in \mathbb{R}_{+}^{4}$. Let $I_{\rho}:=[0, \rho]$ for $\rho>0$. We list our hypotheses as follows.

(H1) $f, g \in C\left([0,1] \times \mathbb{R}_{+}^{8}, \mathbb{R}_{+}\right)$.

(H2) There exist a nonnegative matrix $\left(a_{i j}\right)_{2 \times 4}$ and a constant $c>0$ that

$$
\begin{aligned}
& \left(\begin{array}{l}
f(t, x, y) \\
g(t, x, y)
\end{array}\right) \\
& \quad \geqslant\left(\begin{array}{llll}
a_{11} & a_{12} & a_{13} & a_{14} \\
a_{21} & a_{22} & a_{23} & a_{24}
\end{array}\right)\left(\begin{array}{l}
x_{1}+2 x_{2} \\
x_{3}+2 x_{4} \\
y_{1}+2 y_{4} \\
y_{3}+2 y_{4}
\end{array}\right)-\left(\begin{array}{l}
c \\
c
\end{array}\right)
\end{aligned}
$$

holds for all $(t, x, y) \in[0,1] \times \mathbb{R}_{+}^{8}$, with the matrix

$$
B_{1}:=\left(\begin{array}{cc}
\left(1-2 e^{-1}\right) a_{11}+a_{12}-1 & \left(1-2 e^{-1}\right) a_{13}+a_{14} \\
\left(1-2 e^{-1}\right) a_{21}+a_{22} & \left(1-2 e^{-1}\right) a_{23}+a_{24}-1
\end{array}\right)
$$

being an $\mathbb{R}_{+}^{2}$-monotone matrix.
(H3) There exist $p \in C\left(\mathbb{R}_{+}, \mathbb{R}_{+}\right)$and $q \in C\left(\mathbb{R}_{+}, \mathbb{R}_{+}\right)$such that (1) $p$ is concave; (2) there are two constants $c>0$ and $\mu_{1}>1$ such that

$$
\begin{gathered}
f(t, x, y) \geqslant p\left(y_{3}\right)-c, \quad g(t, x, y) \geqslant q\left(x_{3}\right)-c, \\
\forall(t, x, y) \in[0,1] \times \mathbb{R}_{+}^{8}, \\
p(q(z)) \geqslant \frac{\pi^{4} \mu_{1}}{16} z-c, \quad \forall z \in \mathbb{R}_{+} .
\end{gathered}
$$

(H4) There exist two functions $\Phi_{M}, \Psi_{M} \in C\left(\mathbb{R}_{+}, \mathbb{R}_{+}\right)$, such that

$f(t, x, y) \leqslant \Phi_{M}\left(x_{4}+y_{4}\right), \quad g(t, x, y) \leqslant \Psi_{M}\left(x_{4}+y_{4}\right)$,

for all $(t, x, y) \in[0,1] \times I_{M}^{3} \times \mathbb{R}_{+} \times I_{M}^{3} \times \mathbb{R}_{+}$, and

$$
\int_{0}^{\infty} \frac{\tau \mathrm{d} \tau}{\Phi_{N}(\tau)+\Psi_{N}(\tau)+4 \mu_{0}}>2 M,
$$

where $M:=\max \left\{c_{1}, c_{2}\right\}$, with $c_{1}>0, c_{2}>0, \mu_{0}>0$ being specified in Theorem 11.

(H5) There exist a nonnegative matrix $\left(a_{i j}\right)_{2 \times 4}$ and a constant $r>0$ that

$$
\left(\begin{array}{l}
f(t, x, y) \\
g(t, x, y)
\end{array}\right) \leqslant\left(\begin{array}{llll}
a_{31} & a_{32} & a_{33} & a_{34} \\
a_{41} & a_{42} & a_{43} & a_{44}
\end{array}\right)\left(\begin{array}{l}
x_{1}+2 x_{2} \\
x_{3}+2 x_{4} \\
y_{1}+2 y_{4} \\
y_{3}+2 y_{4}
\end{array}\right)
$$

holds for all $(t, x, y) \in[0,1] \times I_{r}^{8}$, with the matrix

$$
B_{2}:=\left(\begin{array}{cc}
1-a_{11}-a_{12}, & -a_{13}-a_{14} \\
-a_{21}-a_{22}, & 1-a_{23}-a_{24}
\end{array}\right)
$$

being an $\mathbb{R}_{+}^{2}$-monotone matrix.

(H6) There exist a nonnegative matrix $\left(b_{i j}\right)_{2 \times 4}$ and a constant $c>0$ such that

$$
\begin{aligned}
& \left(\begin{array}{l}
f(t, x, y) \\
g(t, x, y)
\end{array}\right) \\
& \quad \leqslant\left(\begin{array}{llll}
b_{11} & b_{12} & b_{13} & b_{14} \\
b_{21} & b_{22} & b_{23} & b_{24}
\end{array}\right)\left(\begin{array}{l}
x_{1}+2 x_{2} \\
x_{3}+2 x_{4} \\
y_{1}+2 y_{4} \\
y_{3}+2 y_{4}
\end{array}\right)+\left(\begin{array}{l}
c \\
c
\end{array}\right)
\end{aligned}
$$

holds for all $(t, x, y) \in[0,1] \times \mathbb{R}_{+}^{8}$, with the matrix

$$
B_{3}:=\left(\begin{array}{cc}
1-b_{11}-b_{12}, & -b_{13}-b_{14} \\
-b_{21}-b_{22}, & 1-b_{23}-b_{24}
\end{array}\right)
$$

being an $\mathbb{R}_{+}^{2}$-monotone matrix.

(H7) There exist a nonnegative matrix $\left(b_{i j}\right)_{2 \times 4}$ and a constant $r>0$ that

$$
\left(\begin{array}{l}
f(t, x, y) \\
g(t, x, y)
\end{array}\right) \geqslant\left(\begin{array}{llll}
b_{31} & b_{32} & b_{33} & b_{34} \\
b_{41} & b_{42} & b_{43} & b_{44}
\end{array}\right)\left(\begin{array}{l}
x_{1}+2 x_{2} \\
x_{3}+2 x_{4} \\
y_{1}+2 y_{4} \\
y_{3}+2 y_{4}
\end{array}\right)
$$


holds for all $(t, x, y) \in[0,1] \times I_{r}^{8}$, with the matrix

$$
B_{4}:=\left(\begin{array}{cc}
\left(1-2 e^{-1}\right) b_{11}+b_{12}-1 & \left(1-2 e^{-1}\right) b_{13}+b_{14} \\
\left(1-2 e^{-1}\right) b_{21}+b_{22} & \left(1-2 e^{-1}\right) b_{23}+b_{24}-1
\end{array}\right)
$$

being an $\mathbb{R}_{+}^{2}$-monotone matrix.

(H8) $f(t, x, y)$ and $g(t, x, y)$ are increasing in $(x, y) \in \mathbb{R}_{+}^{8}$, and there is a constant $\omega>0$ such that

$$
\begin{aligned}
& \int_{0}^{1} f(s, \omega, \omega, \omega, \omega, \omega, \omega, \omega, \omega) \mathrm{d} s<\omega, \\
& \int_{0}^{1} g(s, \omega, \omega, \omega, \omega, \omega, \omega, \omega, \omega) \mathrm{d} s<\omega .
\end{aligned}
$$

Remark 9. A real matrix $B$ is said to be nonnegative if all elements of $B$ are nonnegative.

Remark 10 (see [15, page 112]). A real square matrix $M=$ $\left(m_{i j}\right)_{2 \times 2}$ is called $\mathbb{R}_{+}^{2}$-monotone if, for any column vector $x \in$ $\mathbb{R}^{2}, M x \in \mathbb{R}_{+}^{2} \Rightarrow x \in \mathbb{R}_{+}^{2}$.

Theorem 11. If (H1), (H2), (H4), and (H5) hold, then (1) has at least one positive solution.

Proof. Let

$$
\mathscr{M}_{1}:=\left\{(u, v) \in P^{2}:(u, v)=A(u, v)+\mu(\varphi, \varphi), \mu \geqslant 0\right\},
$$

where $\varphi(t):=t e^{-t}$. We will prove that $\mathscr{M}_{1}$ is bounded. Indeed, if $(u, v) \in \mathscr{M}_{1}$, then there exists $\mu \geqslant 0$ such that $(u, v)=$ $A(u, v)+\mu(\varphi, \varphi)$, which can be written in the form

$$
\begin{aligned}
& u(t)=\int_{0}^{1} k(t, s) f\left(s,(B u)(s),(B u)^{\prime}(s), u(s), u^{\prime}(s),\right. \\
& \left.(B v)(s),(B v)^{\prime}(s), v(s), v^{\prime}(s)\right) \mathrm{d} s \\
& +\mu \varphi(t), \\
& v(t)=\int_{0}^{1} k(t, s) g\left(s,(B u)(s),(B u)^{\prime}(s), u(s), u^{\prime}(s),\right. \\
& \left.(B v)(s),(B v)^{\prime}(s), v(s), v^{\prime}(s)\right) \mathrm{d} s
\end{aligned}
$$

Taking differentiation of the preceding equations twice, we obtain

$$
-u^{\prime \prime}(t)=f\left(t,(B u)(t),(B u)^{\prime}(t), u(t), u^{\prime}(t),\right.
$$$$
\left.(B v)(t),(B v)^{\prime}(t), v(t), v^{\prime}(t)\right)+\mu(2-t) e^{-t},
$$$$
-v^{\prime \prime}(t)=g\left(t,(B u)(t),(B u)^{\prime}(t), u(t), u^{\prime}(t),\right.
$$

$$
\left.(B v)(t),(B v)^{\prime}(t), v(t), v^{\prime}(t)\right)+\mu(2-t) e^{-t},
$$

and thus we have, by $(\mathrm{H} 2)$

$$
\begin{aligned}
& \left(\begin{array}{l}
-u^{\prime \prime}(t) \\
-v^{\prime \prime}(t)
\end{array}\right) \\
& \geqslant\left(\begin{array}{llll}
a_{11} & a_{12} & a_{13} & a_{14} \\
a_{21} & a_{22} & a_{23} & a_{24}
\end{array}\right)\left(\begin{array}{c}
(B u)(t)+2(B u)^{\prime}(t) \\
u(t)+2 u^{\prime}(t) \\
(B v)(t)+2(B v)^{\prime}(t) \\
v(t)+2 v^{\prime}(t)
\end{array}\right)-\left(\begin{array}{l}
c \\
c
\end{array}\right) .
\end{aligned}
$$

Multiply the above by $\varphi(t):=t e^{-t}$ and integrate over $[0,1]$ and use (11) and (12) to obtain

$$
\begin{aligned}
& \left(\begin{array}{c}
\int_{0}^{1}\left(u(t)+2 u^{\prime}(t)\right) t e^{t} \mathrm{~d} t \\
\int_{0}^{1}\left(v(t)+2 v^{\prime}(t)\right) t e^{t} \mathrm{~d} t
\end{array}\right) \\
& \geqslant\left(\begin{array}{llll}
a_{11} & a_{12} & a_{13} & a_{14} \\
a_{21} & a_{22} & a_{23} & a_{24}
\end{array}\right)\left(\begin{array}{c}
\int_{0}^{1} u(t) t e^{t} \mathrm{~d} t \\
\int_{0}^{1}\left(u(t)+2 u^{\prime}(t)\right) t e^{t} \mathrm{~d} t \\
\int_{0}^{1} v(t) t e^{t} \mathrm{~d} t \\
\int_{0}^{1}\left(v(t)+2 v^{\prime}(t)\right) t e^{t} \mathrm{~d} t
\end{array}\right) \\
& -\left(\begin{array}{l}
c \\
c
\end{array}\right) .
\end{aligned}
$$

It is readily seen that, for any $u, v \in \mathscr{M}_{1}, u^{\prime}$ and $v^{\prime}$ are decreasing on $[0,1]$. By (13), we obtain

$$
\begin{aligned}
& \left(\begin{array}{c}
\int_{0}^{1}\left(u(t)+2 u^{\prime}(t)\right) t e^{t} \mathrm{~d} t \\
\int_{0}^{1}\left(v(t)+2 v^{\prime}(t)\right) t e^{t} \mathrm{~d} t
\end{array}\right) \\
& \geqslant\left(\begin{array}{ll}
\left(1-2 e^{-1}\right) a_{11}+a_{12} & \left(1-2 e^{-1}\right) a_{13}+a_{14} \\
\left(1-2 e^{-1}\right) a_{21}+a_{22} & \left(1-2 e^{-1}\right) a_{23}+a_{24}
\end{array}\right) \\
& \times\left(\begin{array}{c}
\int_{0}^{1}\left(u(t)+2 u^{\prime}(t)\right) t e^{t} \mathrm{~d} t \\
\int_{0}^{1}\left(v(t)+2 v^{\prime}(t)\right) t e^{t} \mathrm{~d} t
\end{array}\right)-\left(\begin{array}{l}
c \\
c
\end{array}\right)
\end{aligned}
$$

so that

$$
B_{1}\left(\begin{array}{l}
\int_{0}^{1}\left(u(t)+2 u^{\prime}(t)\right) t e^{t} \mathrm{~d} t \\
\int_{0}^{1}\left(v(t)+2 v^{\prime}(t)\right) t e^{t} \mathrm{~d} t
\end{array}\right) \leqslant\left(\begin{array}{l}
c \\
c
\end{array}\right) .
$$


Since $B_{1}$ is an $\mathbb{R}_{+}^{2}$-monotone matrix, we have

$$
\left(\begin{array}{c}
\int_{0}^{1}\left(u(t)+2 u^{\prime}(t)\right) t e^{t} \mathrm{~d} t \\
\int_{0}^{1}\left(v(t)+2 v^{\prime}(t)\right) t e^{t} \mathrm{~d} t
\end{array}\right) \leqslant B_{1}^{-1}\left(\begin{array}{l}
c \\
c
\end{array}\right):=\left(\begin{array}{l}
c_{1} \\
c_{2}
\end{array}\right) .
$$

Now Lemma 4 implies

$$
\|u\|_{0}=u(1) \leqslant c_{1}, \quad\|v\|_{0}=v(1) \leqslant c_{2}, \quad \forall(u, v) \in \mathscr{M}_{1} .
$$

Furthermore, these estimates lead to

$$
\begin{gathered}
\|(B u)(t)\|_{0}=(B u)(1)=\int_{0}^{1} s u(s) \mathrm{d} s \leqslant c_{1}, \\
\|(B v)(t)\|_{0}=(B v)(1)=\int_{0}^{1} s v(s) \mathrm{d} s \leqslant c_{2}, \\
\left\|(B u)^{\prime}(t)\right\|_{0}=(B u)^{\prime}(0)=\int_{0}^{1} u(s) \mathrm{d} s \leqslant c_{1}, \\
\left\|(B v)^{\prime}(t)\right\|_{0}=(B v)^{\prime}(0)=\int_{0}^{1} v(s) \mathrm{d} s \leqslant c_{2},
\end{gathered}
$$

for all $(u, v) \in \mathscr{M}_{1}$. Let

$$
\begin{aligned}
\Pi:=\{\mu \geqslant 0: & \text { there exists some }(u, v) \in P^{2}, \\
& \text { such that }(u, v)=A(u, v)+\mu(\varphi, \varphi)\} .
\end{aligned}
$$

Equation (42) implies that $\mu_{0}:=\sup \Pi<\infty$. Let $M:=$ $\max \left\{c_{1}, c_{2}\right\}$. By $(\mathrm{H} 4)$ again, there are two functions $\Phi_{M}, \Psi_{M} \in$ $C\left(\mathbb{R}_{+}, \mathbb{R}_{+}\right)$, for all $(u, v) \in \mathscr{M}_{1}$, such that

$$
\begin{aligned}
& f\left(t,(B u)(t),(B u)^{\prime}(t), u(t), u^{\prime}(t),\right. \\
& \left.(B v)(t),(B v)^{\prime}(t), v(t), v^{\prime}(t)\right) \leqslant \Phi_{M}\left(u^{\prime}(t)+v^{\prime}(t)\right), \\
& g\left(t,(B u)(t),(B u)^{\prime}(t), u(t), u^{\prime}(t),\right. \\
& \left.(B v)(t),(B v)^{\prime}(t), v(t), v^{\prime}(t)\right) \leqslant \Psi_{M}\left(u^{\prime}(t)+v^{\prime}(t)\right) .
\end{aligned}
$$

Thus we have

$$
\begin{aligned}
-u^{\prime \prime}(t)= & f\left(t,(B u)(t),(B u)^{\prime}(t), u(t), u^{\prime}(t),\right. \\
& \left.(B v)(t),(B v)^{\prime}(t), v(t), v^{\prime}(t)\right)+\mu(2-t) e^{-t} \\
\leqslant & \Phi_{M}\left(u^{\prime}(t)+v^{\prime}(t)\right)+\mu(2-t) e^{-t} \\
\leqslant & \Phi_{M}\left(u^{\prime}(t)+v^{\prime}(t)\right)+2 \mu_{0}, \\
-v^{\prime \prime}(t)= & g\left(t,(B u)(t),(B u)^{\prime}(t), u(t), u^{\prime}(t),\right. \\
& \left.(B v)(t),(B v)^{\prime}(t), v(t), v^{\prime}(t)\right)+\mu(2-t) e^{-t} \\
\leqslant & \Psi_{M}\left(u^{\prime}(t)+v^{\prime}(t)\right)+\mu(2-t) e^{-t} \\
\leqslant & \Psi_{M}\left(u^{\prime}(t)+v^{\prime}(t)\right)+2 \mu_{0} .
\end{aligned}
$$

This implies, for all $(u, v) \in \mathscr{M}_{1}$

$$
\begin{aligned}
-\left(u^{\prime \prime}(t)+v^{\prime \prime}(t)\right)\left(u^{\prime}(t)\right. & \left.+v^{\prime}(t)\right) \\
\leqslant\left(u^{\prime}(t)+v^{\prime}(t)\right) & \left(\Phi_{N}\left(u^{\prime}(t)+v^{\prime}(t)\right)\right. \\
& \left.+\Psi_{N}\left(u^{\prime}(t)+v^{\prime}(t)\right)+4 \mu_{0}\right),
\end{aligned}
$$

and, in turn,

$$
\begin{gathered}
\int_{0}^{u^{\prime}(0)+v^{\prime}(0)} \frac{\tau \mathrm{d} \tau}{\Phi_{N}(\tau)+\Psi_{N}(\tau)+4 \mu_{0}} \\
\quad \leqslant \int_{0}^{1}\left(u^{\prime}(t)+v^{\prime}(t)\right) \mathrm{d} t=u(1)+v(1) \leqslant 2 M .
\end{gathered}
$$

By (H4) again, there exists a constant $N_{1}>0$ such that

$$
\left\|u^{\prime}+v^{\prime}\right\|_{0}=u^{\prime}(0)+v^{\prime}(0) \leqslant N_{1}, \quad \forall(u, v) \in \mathscr{M}_{1} .
$$

This means that $\mathscr{M}_{1}$ is bounded. Taking $R>\sup \{\|(u, v)\|$ : $\left.(u, v) \in \mathscr{M}_{1}\right\}$, we have

$$
(u, v) \neq A(u, v)+\mu(\varphi, \varphi), \quad \forall(u, v) \in \partial \Omega_{R} \cap P^{2}, \mu \geqslant 0 .
$$

Now Lemma 6 yields

$$
i\left(A, \Omega_{R} \cap P^{2}, P^{2}\right)=0 .
$$

Let

$$
\mathscr{M}_{2}:=\left\{(u, v) \in \bar{\Omega}_{r} \cap P^{2}:(u, v)=\lambda A(u, v), \lambda \in[0,1]\right\},
$$

where $r>0$ is given in (H5). We are in the position to prove $\mathscr{M}_{2}=\{0\}$. Indeed, if $(u, v) \in \mathscr{M}_{2}$, then $(u, v)=\lambda A(u, v)$ for some $\lambda \in[0,1]$, which can be written componentwise as

$$
\begin{array}{r}
u(t)=\lambda \int_{0}^{1} k(t, s) f\left(s,(B u)(s),(B u)^{\prime}(s),\right. \\
u(s), u^{\prime}(s),(B v)(s), \\
\left.(B v)^{\prime}(s), v(s), v^{\prime}(s)\right) \mathrm{d} s, \\
v(t)=\lambda \int_{0}^{1} k(t, s) g\left(s,(B u)(s),(B u)^{\prime}(s),\right. \\
u(s), u^{\prime}(s),(B v)(s), \\
\left.(B v)^{\prime}(s), v(s), v^{\prime}(s)\right) \mathrm{d} s .
\end{array}
$$

Taking differentiation of the preceding equations twice, we obtain

$$
\begin{array}{r}
-u^{\prime \prime}(t)=\lambda f\left(t,(B u)(t),(B u)^{\prime}(t), u(t), u^{\prime}(t),\right. \\
\left.(B v)(t),(B v)^{\prime}(t), v(t), v^{\prime}(t)\right), \\
-v^{\prime \prime}(t)=\lambda g\left(t,(B u)(t),(B u)^{\prime}(t), u(t), u^{\prime}(t),\right. \\
\left.(B v)(t),(B v)^{\prime}(t), v(t), v^{\prime}(t)\right) .
\end{array}
$$


By (H5), we have

$$
\left(\begin{array}{c}
-u^{\prime \prime}(t) \\
-v^{\prime \prime}(t)
\end{array}\right) \leqslant\left(\begin{array}{llll}
a_{31} & a_{32} & a_{33} & a_{34} \\
a_{41} & a_{42} & a_{43} & a_{44}
\end{array}\right)\left(\begin{array}{c}
(B u)(t)+2(B u)^{\prime}(t) \\
u(t)+2 u^{\prime}(t) \\
(B v)(t)+2(B v)^{\prime}(t) \\
v(t)+2 v^{\prime}(t)
\end{array}\right)
$$

Multiply the above by $\varphi(t):=t e^{-t}$ and use (11) and (12) to obtain

$$
\begin{aligned}
& \left(\begin{array}{c}
\int_{0}^{1}\left(u(t)+2 u^{\prime}(t)\right) t e^{t} \mathrm{~d} t \\
\int_{0}^{1}\left(v(t)+2 v^{\prime}(t)\right) t e^{t} \mathrm{~d} t
\end{array}\right) \\
& \leqslant\left(\begin{array}{llll}
a_{31} & a_{32} & a_{33} & a_{34} \\
a_{41} & a_{42} & a_{43} & a_{44}
\end{array}\right)\left(\begin{array}{c}
\int_{0}^{1} u(t) t e^{t} \mathrm{~d} t \\
\int_{0}^{1}\left(u(t)+2 u^{\prime}(t)\right) t e^{t} \mathrm{~d} t \\
\int_{0}^{1} v(t) t e^{t} \mathrm{~d} t \\
\int_{0}^{1}\left(v(t)+2 v^{\prime}(t)\right) t e^{t} \mathrm{~d} t
\end{array}\right) \\
& \leqslant\left(\begin{array}{ll}
a_{31}+a_{32} & a_{33}+a_{34} \\
a_{41}+a_{42} & a_{43}+a_{44}
\end{array}\right)\left(\begin{array}{c}
\int_{0}^{1}\left(u(t)+2 u^{\prime}(t)\right) t e^{t} \mathrm{~d} t \\
\int_{0}^{1}\left(v(t)+2 v^{\prime}(t)\right) t e^{t} \mathrm{~d} t
\end{array}\right),
\end{aligned}
$$

so that

$$
B_{2}\left(\begin{array}{c}
\int_{0}^{1}\left(u(t)+2 u^{\prime}(t)\right) t e^{t} \mathrm{~d} t \\
\int_{0}^{1}\left(v(t)+2 v^{\prime}(t)\right) t e^{t} \mathrm{~d} t
\end{array}\right) \leqslant\left(\begin{array}{l}
0 \\
0
\end{array}\right)
$$

Since $B_{2}$ is an $\mathbb{R}_{+}^{2}$-monotone matrix, it follows that $\int_{0}^{1}(u(t)+$ $\left.2 u^{\prime}(t)\right) t e^{t} \mathrm{~d} t=0$ and $\int_{0}^{1}\left(v(t)+2 v^{\prime}(t)\right) t e^{t} \mathrm{~d} t=0$, so that $u=v \equiv$ 0 . This proves $\mathscr{M}_{2}=\{0\}$, as required. Consequently,

$$
(u, v) \neq \lambda A(u, v), \quad \forall(u, v) \in \partial \Omega_{r} \cap P^{2}, \lambda \in[0,1] .
$$

Now Lemma 7 yields

$$
i\left(A, \Omega_{r} \cap P^{2}, P^{2}\right)=1 .
$$

Combining (51) and (59) we arrive at

$$
i\left(A,\left(\Omega_{R} \backslash \bar{\Omega}_{r}\right) \cap P^{2}, P^{2}\right)=0-1=-1 .
$$

Therefore $A$ has at least one fixed point on $\left(\Omega_{R} \backslash \bar{\Omega}_{r}\right) \cap P^{2}$. Thus (1) has at least one positive solution, which completes the proof.

Theorem 12. If (H1), (H3), (H4), and (H5) are satisfied, then (1) has at least one positive solution.
Proof. By (H3), we obtain

$$
\begin{aligned}
& A_{1}(u, v)(t) \geqslant \int_{0}^{1} k(t, s) p(v(s)) \mathrm{d} s-\widehat{c}_{1}, \\
& A_{2}(u, v)(t) \geqslant \int_{0}^{1} k(t, s) q(u(s)) \mathrm{d} s-\widehat{c}_{1}
\end{aligned}
$$

for all $(u, v) \in P^{2}, t \in[0,1]$. We claim that the set

$$
\mathscr{M}_{3}:=\left\{(u, v) \in P^{2}:(u, v)=A(u, v)+\lambda(\varphi, \varphi), \lambda \geqslant 0\right\}
$$

is bounded, where $\varphi(t):=t e^{-t}$. Indeed, if $(u, v) \in \mathscr{M}_{3}$, then there exists a constant $\lambda \geqslant 0$ such that $(u, v)=A(u, v)+$ $\lambda(\varphi, \varphi)$, which can be written in the form

$$
\begin{aligned}
u(t)= & \int_{0}^{1} k(t, s) f\left(s,(B u)(s),(B u)^{\prime}(s), u(s), u^{\prime}(s),\right. \\
& \left.(B v)(s),(B v)^{\prime}(s), v(s), v^{\prime}(s)\right) \mathrm{d} s \\
& +\lambda \varphi(t), \\
v(t)= & \int_{0}^{1} k(t, s) g\left(s,(B u)(s),(B u)^{\prime}(s), u(s), u^{\prime}(s),\right. \\
& \left.\quad(B v)(s),(B v)^{\prime}(s), v(s), v^{\prime}(s)\right) \mathrm{d} s \\
&
\end{aligned}
$$

By (H3) and (61), we have

$$
\begin{aligned}
& u(t) \geqslant \int_{0}^{1} k(t, s) p(v(s)) \mathrm{d} s-\widehat{c}_{1}, \\
& v(t) \geqslant \int_{0}^{1} k(t, s) q(u(s)) \mathrm{d} s-\widehat{c}_{1}
\end{aligned}
$$

for all $t \in[0,1]$. The nonnegativity and concavity of $p$ imply $\lim _{y \rightarrow \infty} p(y) / y \geqslant 0$. Now Lemma 8 and Jensen's inequality imply

$$
\begin{aligned}
p(v(t)) & \geqslant p\left(v(t)+\widehat{c}_{1}\right)-p\left(\widehat{c}_{1}\right) \\
& \geqslant \int_{0}^{1} k(t, s) p(q(u(s))) \mathrm{d} s-p\left(\widehat{c}_{1}\right) .
\end{aligned}
$$

This, together with (64) and (H3), implies

$$
\begin{aligned}
u(t) & \geqslant \int_{0}^{1} k(t, s)\left[\int_{0}^{1} k(s, \tau) p(q(u(\tau))) \mathrm{d} \tau-p\left(\widehat{c}_{1}\right)\right] \mathrm{d} s-\widehat{c}_{1} \\
& \geqslant \int_{0}^{1} \int_{0}^{1} k(t, s) k(s, \tau) p(q(u(\tau))) \mathrm{d} s \mathrm{~d} \tau-\widehat{c}_{2} \\
& \geqslant \int_{0}^{1} \int_{0}^{1} k(t, s) k(s, \tau)\left[\frac{\pi^{4} \mu_{1}}{16} u(\tau)-c\right] \mathrm{d} s \mathrm{~d} \tau-\widehat{c}_{2} \\
& \geqslant \frac{\pi^{4} \mu_{1}}{16} \int_{0}^{1} \int_{0}^{1} k(t, s) k(s, \tau) u(\tau) \mathrm{d} s \mathrm{~d} \tau-\widehat{c}_{3} .
\end{aligned}
$$


Multiply the last inequality by $\varphi(t):=t e^{-t}$ and integrate over $[0,1]$ and use $(7)$ twice to obtain

$$
\begin{aligned}
\int_{0}^{1} \varphi(t) & u(t) \mathrm{d} t \\
\geqslant & \frac{\pi^{4} \mu_{1}}{16} \int_{0}^{1} \int_{0}^{1} \int_{0}^{1} \varphi(t) k(t, s) k(s, \tau) u(\tau) \mathrm{d} t \mathrm{~d} s \mathrm{~d} \tau \\
& -\frac{2 \widehat{c}_{3}}{\pi} \\
= & \mu_{1} \int_{0}^{1} \varphi(t) u(t) \mathrm{d} t-\frac{2 \widehat{c}_{3}}{\pi},
\end{aligned}
$$

so that

$$
\int_{0}^{1} \varphi(t) u(t) \mathrm{d} t \leqslant \frac{2 \widehat{c}_{3}}{\pi\left(\mu_{1}-1\right)} .
$$

By Lemma 5, we have

$$
\|u\|_{0}=u(1) \leqslant \frac{2 \widehat{c}_{3}}{\pi \varrho(\varphi)\left(\mu_{1}-1\right)}=\frac{\widehat{c}_{3} \pi}{2\left(\mu_{1}-1\right)} .
$$

Multiply the first inequality of (64) by $\varphi(t)$, integrate over $[0,1]$, and use ( 7$)$ to obtain

$$
\int_{0}^{1} u(t) \varphi(t) \mathrm{d} t \geqslant \frac{4}{\pi^{2}} \int_{0}^{1} p(v(t)) \varphi(t) \mathrm{d} t-\frac{2}{\pi} \widehat{c}_{1} .
$$

This, along with (68), implies

$$
\begin{aligned}
\int_{0}^{1} p(v(t)) \varphi(t) \mathrm{d} t & \leqslant \frac{\pi^{2}}{4}\left(\frac{2 \widehat{c}_{1}}{\pi}+\int_{0}^{1} u(t) \varphi(t) \mathrm{d} t\right) \\
& \leqslant \frac{\widehat{c}_{1} \pi}{2}+\frac{\widehat{c}_{3} \pi}{2\left(\mu_{1}-1\right)}
\end{aligned}
$$

By Lemma 5, we have

$$
\begin{aligned}
\|v\|_{0} & =v(1) \leqslant \frac{1}{\varrho(\varphi)} \int_{0}^{1} v(t) \varphi(t) \mathrm{d} t \\
& =\frac{\|v\|_{0}}{\varrho(\varphi) p\left(\|v\|_{0}\right)} \int_{0}^{1} \varphi(t) \frac{v(t)}{\|v\|_{0}} p\left(\|v\|_{0}\right) \mathrm{d} t \\
& \leqslant \frac{\|v\|_{0}}{\varrho(\varphi) p\left(\|v\|_{0}\right)} \int_{0}^{1} \varphi(t) p(v(t)) \mathrm{d} t,
\end{aligned}
$$

so that

$$
\begin{aligned}
p\left(\|v\|_{0}\right) & \leqslant \frac{1}{\varrho(\varphi)} \int_{0}^{1} \varphi(t) p(v(t)) \mathrm{d} t \\
& \leqslant \frac{\widehat{c}_{1} \pi^{3}}{8}+\frac{\widehat{c}_{3} \pi^{4}}{16\left(\mu_{1}-1\right)} .
\end{aligned}
$$

(H3) implies that $p$ is strictly increasing and $\lim _{x \rightarrow \infty} p(x)=$ $\infty$ (see Lemma 8). Consequently, there exists $\widehat{c}_{4}>0$ such that

$$
\|v\|_{0} \leqslant \widehat{c}_{4}
$$

Let $N:=\max \left\{\widehat{c}_{3} \pi / 2\left(\mu_{1}-1\right), \widehat{c}_{4}\right\}$. Then

$$
\|u\|_{0} \leqslant N, \quad\|v\|_{0} \leqslant N, \quad \forall(u, v) \in \mathscr{M}_{3} .
$$

This establishes the a priori bound of $\mathscr{M}_{3}$ for $\|(u, v)\|_{0}$. Now it remains to derive the a priori bound of $\mathscr{M}_{3}$ for $\left\|\left(u^{\prime}, v^{\prime}\right)\right\|_{0}$, which is similar to the derivation of the a priori bound of $\mathscr{M}_{1}$ for $\left\|\left(u^{\prime}, v^{\prime}\right)\right\|_{0}$ in Theorem 11 . This means that $\mathscr{M}_{3}$ is bounded. Taking $R>\sup \left\{\|(u, v)\|:(u, v) \in \mathscr{M}_{3}\right\}$, we have

$$
(u, v) \neq A(u, v)+\mu(\varphi, \varphi), \quad \forall(u, v) \in \partial \Omega_{R} \cap P^{2}, \mu \geqslant 0 .
$$

Now Lemma 6 yields

$$
i\left(A, \Omega_{R} \cap P^{2}, P^{2}\right)=0 .
$$

Note that (H1) and (H5) imply (59) holds (see the proof of Theorem 11). Combining (77) and (59) we arrive at

$$
i\left(A,\left(\Omega_{R} \backslash \bar{\Omega}_{r}\right) \cap P^{2}, P^{2}\right)=0-1=-1 .
$$

Therefore $A$ has at least one fixed point on $\left(\Omega_{R} \backslash \bar{\Omega}_{r}\right) \cap P^{2}$. Thus (1) has at least one positive solution, which completes the proof.

Theorem 13. If (H1), (H6), and (H7) are satisfied, then (1) has at least one positive solution.

Proof. Let

$$
\mathscr{M}_{4}:=\left\{(u, v) \in P^{2}:(u, v)=\lambda A(u, v), \lambda \in[0,1]\right\} .
$$

We will prove that $\mathscr{M}_{4}$ is bounded. Indeed, if $(u, v) \in \mathscr{M}_{4}$, then $(u, v) \in P^{2}$ and $(u, v)=\lambda A(u, v)$, for some $\lambda \in[0,1]$, which can be written componentwise as

$$
\begin{array}{r}
u(t)=\lambda \int_{0}^{1} k(t, s) f\left(s,(B u)(s),(B u)^{\prime}(s), u(s), u^{\prime}(s),\right. \\
\left.(B v)(s),(B v)^{\prime}(s), v(s), v^{\prime}(s)\right) \mathrm{d} s \\
v(t)=\lambda \int_{0}^{1} k(t, s) g\left(s,(B u)(s),(B u)^{\prime}(s), u(s), u^{\prime}(s),\right. \\
\left.(B v)(s),(B v)^{\prime}(s), v(s), v^{\prime}(s)\right) \mathrm{d} s .
\end{array}
$$

Taking differentiation of the preceding equations twice, we obtain

$$
\begin{gathered}
-u^{\prime \prime}(t)=\lambda f\left(t,(B u)(t),(B u)^{\prime}(t), u(t), u^{\prime}(t),(B v)(t),\right. \\
\left.(B v)^{\prime}(t), v(t), v^{\prime}(t)\right), \\
-v^{\prime \prime}(t)=\lambda g\left(t,(B u)(t),(B u)^{\prime}(t), u(t), u^{\prime}(t),(B v)(t),\right. \\
\left.(B v)^{\prime}(t), v(t), v^{\prime}(t)\right) .
\end{gathered}
$$


By (H6), we have

$$
\begin{aligned}
& \left(\begin{array}{c}
-u^{\prime \prime}(t) \\
-v^{\prime \prime}(t)
\end{array}\right) \\
& \quad \leqslant\left(\begin{array}{llll}
b_{11} & b_{12} & b_{13} & b_{14} \\
b_{21} & b_{22} & b_{23} & b_{24}
\end{array}\right)\left(\begin{array}{c}
(B u)(t)+2(B u)^{\prime}(t) \\
u(t)+2 u^{\prime}(t) \\
(B v)(t)+2(B v)^{\prime}(t) \\
v(t)+2 v^{\prime}(t)
\end{array}\right)+\left(\begin{array}{c}
c \\
c
\end{array}\right) .
\end{aligned}
$$

Multiply the above by $\varphi(t):=t e^{-t}$ and use (11) and to obtain (12) :

$$
\begin{aligned}
& \left(\begin{array}{c}
\int_{0}^{1}\left(u(t)+2 u^{\prime}(t)\right) t e^{t} \mathrm{~d} t \\
\int_{0}^{1}\left(v(t)+2 v^{\prime}(t)\right) t e^{t} \mathrm{~d} t
\end{array}\right) \\
& \leqslant\left(\begin{array}{llll}
b_{11} & b_{12} & b_{13} & b_{14} \\
b_{21} & b_{22} & b_{23} & b_{24}
\end{array}\right)\left(\begin{array}{c}
\int_{0}^{1} u(t) t e^{t} \mathrm{~d} t \\
\int_{0}^{1}\left(u(t)+2 u^{\prime}(t)\right) t e^{t} \mathrm{~d} t \\
\int_{0}^{1} v(t) t e^{t} \mathrm{~d} t \\
\int_{0}^{1}\left(v(t)+2 v^{\prime}(t)\right) t e^{t} \mathrm{~d} t
\end{array}\right) \\
& +\left(\begin{array}{l}
c \\
c
\end{array}\right) \\
& \leqslant\left(\begin{array}{ll}
b_{11}+b_{12} & b_{13}+b_{14} \\
b_{21}+b_{22} & b_{23}+b_{24}
\end{array}\right)\left(\begin{array}{l}
\int_{0}^{1}\left(u(t)+2 u^{\prime}(t)\right) t e^{t} \mathrm{~d} t \\
\int_{0}^{1}\left(v(t)+2 v^{\prime}(t)\right) t e^{t} \mathrm{~d} t
\end{array}\right) \\
& +\left(\begin{array}{l}
c \\
c
\end{array}\right)
\end{aligned}
$$

so that

$$
B_{3}\left(\begin{array}{c}
\int_{0}^{1}\left(u(t)+2 u^{\prime}(t)\right) t e^{t} \mathrm{~d} t \\
\int_{0}^{1}\left(v(t)+2 v^{\prime}(t)\right) t e^{t} \mathrm{~d} t
\end{array}\right) \leqslant\left(\begin{array}{l}
c \\
c
\end{array}\right)
$$

Since $B_{3}$ is an $\mathbb{R}_{+}^{2}$-monotone matrix, we have

$$
\left(\begin{array}{c}
\int_{0}^{1}\left(u(t)+2 u^{\prime}(t)\right) t e^{t} \mathrm{~d} t \\
\int_{0}^{1}\left(v(t)+2 v^{\prime}(t)\right) t e^{t} \mathrm{~d} t
\end{array}\right) \leqslant B_{3}^{-1}\left(\begin{array}{l}
c \\
c
\end{array}\right):=\left(\begin{array}{l}
c_{3} \\
c_{4}
\end{array}\right) .
$$

Now Lemma 4 implies

$$
\|u\|_{0}=u(1) \leqslant c_{3}, \quad\|v\|_{0}=v(1) \leqslant c_{4}, \quad \forall(u, v) \in \mathscr{M}_{1} .
$$

Furthermore, these estimates lead to

$$
\begin{gathered}
\|(B u)(t)\|_{0}=(B u)(1)=\int_{0}^{1} s u(s) \mathrm{d} s \leqslant c_{3}, \\
\|(B v)(t)\|_{0}=(B v)(1)=\int_{0}^{1} s v(s) \mathrm{d} s \leqslant c_{4}, \\
\left\|(B u)^{\prime}(t)\right\|_{0}=(B u)^{\prime}(0)=\int_{0}^{1} u(s) \mathrm{d} s \leqslant c_{3}, \\
\left\|(B v)^{\prime}(t)\right\|_{0}=(B v)^{\prime}(0)=\int_{0}^{1} v(s) \mathrm{d} s \leqslant c_{4} .
\end{gathered}
$$

Therefore, for all $(u, v) \in \mathscr{M}_{4}$, we have

$$
\begin{aligned}
& \left(\begin{array}{l}
-u^{\prime \prime}(t) \\
-v^{\prime \prime}(t)
\end{array}\right) \\
& \leqslant\left(\begin{array}{llll}
b_{11} & b_{12} & b_{13} & b_{14} \\
b_{21} & b_{22} & b_{23} & b_{24}
\end{array}\right)\left(\begin{array}{c}
(B u)(t)+2(B u)^{\prime}(t) \\
u(t)+2 u^{\prime}(t) \\
(B v)(t)+2(B v)^{\prime}(t) \\
v(t)+2 v^{\prime}(t)
\end{array}\right) \\
& +\left(\begin{array}{l}
c \\
c
\end{array}\right) \\
& \leqslant\left(\begin{array}{llll}
3 b_{11}+b_{12} & 3 b_{13}+b_{14} & 2 b_{12} & 2 b_{14} \\
3 b_{21}+b_{22} & 3 b_{23}+b_{24} & 2 b_{22} & 2 b_{24}
\end{array}\right)\left(\begin{array}{c}
c_{3} \\
c_{4} \\
u^{\prime}(t) \\
v^{\prime}(t)
\end{array}\right) \\
& +\left(\begin{array}{l}
c \\
c
\end{array}\right) .
\end{aligned}
$$

Applying Lemma 3 in [16], we may establish the boundedness of $u^{\prime}, v^{\prime}$ of $\mathscr{M}_{4}$. Taking $R>\sup \left\{\|(u, v)\|:(u, v) \in \mathscr{M}_{4}\right\}$, we have

$$
(u, v) \neq \lambda A(u, v), \quad \forall(u, v) \in \partial \Omega_{R} \cap(P \times P), \lambda \in[0,1] .
$$

Lemma 7 yields

$$
i\left(A, \Omega_{R} \cap P^{2}, P^{2}\right)=1 .
$$

Let

$$
\begin{aligned}
& \mathscr{M}_{5}:=\left\{(u, v) \in \bar{\Omega}_{r_{2}} \cap P^{2}:\right. \\
&(u, v)=A(u, v)+\lambda(\varphi, \varphi), \lambda \geqslant 0\},
\end{aligned}
$$

where $\varphi(t):=t e^{-t}$ and $r>0$ is given in (H7). Next we will prove $\mathscr{M}_{5} \subset\{0\}$. Indeed, if $(u, v) \in \mathscr{M}_{5}$, then there exists 
$\lambda \geqslant 0$ such that $(u, v)=A(u, v)+\lambda(\varphi, \varphi)$, which can be written in the form

$$
\begin{array}{r}
u(t)=\int_{0}^{1} k(t, s) f\left(s,(B u)(s),(B u)^{\prime}(s), u(s), u^{\prime}(s),\right. \\
\left.(B v)(s),(B v)^{\prime}(s), v(s), v^{\prime}(s)\right) \mathrm{d} s
\end{array}
$$

$$
\begin{gathered}
+\lambda \varphi(t), \\
v(t)=\int_{0}^{1} k(t, s) g\left(s,(B u)(s),(B u)^{\prime}(s), u(s), u^{\prime}(s),\right. \\
\left.(B v)(s),(B v)^{\prime}(s), v(s), v^{\prime}(s)\right) \mathrm{d} s
\end{gathered}
$$$$
+\lambda \varphi(t) .
$$

Taking differentiation of the preceding equations twice, we obtain

$$
\begin{aligned}
&-u^{\prime \prime}(t)= f\left(t,(B u)(t),(B u)^{\prime}(t), u(t), u^{\prime}(t),\right. \\
&\left.(B v)(t),(B v)^{\prime}(t), v(t), v^{\prime}(t)\right)+\lambda(2-t) e^{-t}, \\
&-v^{\prime \prime}(t)= g\left(t,(B u)(t),(B u)^{\prime}(t), u(t), u^{\prime}(t),\right. \\
&\left.(B v)(t),(B v)^{\prime}(t), v(t), v^{\prime}(t)\right) \\
&+\lambda(2-t) e^{-t} .
\end{aligned}
$$

By (H7), we have

$$
\begin{aligned}
& \left(\begin{array}{l}
-u^{\prime \prime}(t) \\
-v^{\prime \prime}(t)
\end{array}\right) \\
& \quad \geqslant\left(\begin{array}{llll}
b_{31} & b_{32} & b_{33} & b_{34} \\
b_{41} & b_{42} & b_{43} & b_{44}
\end{array}\right)\left(\begin{array}{c}
(B u)(t)+2(B u)^{\prime}(t) \\
u(t)+2 u^{\prime}(t) \\
(B v)(t)+2(B v)^{\prime}(t) \\
v(t)+2 v^{\prime}(t)
\end{array}\right) .
\end{aligned}
$$

Multiply the above by $\varphi(t):=t e^{-t}$ and integrate over $[0,1]$ and use (11) and (13). Notice that $u^{\prime}, v^{\prime}$ are decreasing on $[0,1]$; we obtain

$$
\begin{aligned}
& \left(\begin{array}{l}
\int_{0}^{1}\left(u(t)+2 u^{\prime}(t)\right) t e^{t} \mathrm{~d} t \\
\int_{0}^{1}\left(v(t)+2 v^{\prime}(t)\right) t e^{t} \mathrm{~d} t
\end{array}\right) \\
& \geqslant\left(\begin{array}{llll}
b_{31} & b_{32} & b_{33} & b_{34} \\
b_{41} & b_{42} & b_{43} & b_{44}
\end{array}\right)\left(\begin{array}{c}
\int_{0}^{1} u(t) t e^{t} \mathrm{~d} t \\
\int_{0}^{1}\left(\begin{array}{c}
\left.u(t)+2 u^{\prime}(t)\right) t e^{t} \mathrm{~d} t \\
\int_{0}^{1} v(t) t e^{t} \mathrm{~d} t \\
\int_{0}^{1}\left(v(t)+2 v^{\prime}(t)\right) t e^{t} \mathrm{~d} t
\end{array}\right)
\end{array}\right.
\end{aligned}
$$

$$
\begin{aligned}
& \geqslant\left(\begin{array}{l}
\left(1-2 e^{-1}\right) b_{31}+b_{32}\left(1-2 e^{-1}\right) b_{33}+b_{34} \\
\left(1-2 e^{-1}\right) b_{41}+b_{42}\left(1-2 e^{-1}\right) b_{43}+b_{44}
\end{array}\right) \\
& \times\left(\begin{array}{c}
\int_{0}^{1}\left(u(t)+2 u^{\prime}(t)\right) t e^{t} \mathrm{~d} t \\
\int_{0}^{1}\left(v(t)+2 v^{\prime}(t)\right) t e^{t} \mathrm{~d} t
\end{array}\right),
\end{aligned}
$$

so that

$$
B_{4}\left(\begin{array}{c}
\int_{0}^{1}\left(u(t)+2 u^{\prime}(t)\right) t e^{t} \mathrm{~d} t \\
\int_{0}^{1}\left(v(t)+2 v^{\prime}(t)\right) t e^{t} \mathrm{~d} t
\end{array}\right) \leqslant\left(\begin{array}{l}
0 \\
0
\end{array}\right) .
$$

Since $B_{4}$ is an $\mathbb{R}_{+}^{2}$-monotone matrix, it follows that $\int_{0}^{1}(u(t)+$ $\left.2 u^{\prime}(t)\right) t e^{t} \mathrm{~d} t=0$ and $\int_{0}^{1}\left(v(t)+2 v^{\prime}(t)\right) t e^{t} \mathrm{~d} t=0$, so that $u=v \equiv$ 0 . This proves $\mathscr{M}_{5} \subset\{0\}$, as required. Consequently,

$$
(u, v) \neq A(u, v)+\lambda(\varphi, \varphi), \quad \forall(u, v) \in \partial \Omega_{r_{2}} \cap P^{2}, \lambda \geqslant 0 .
$$

Now Lemma 6 yields

$$
i\left(A, \Omega_{r_{2}} \cap P^{2}, P^{2}\right)=0 .
$$

Combining (90) and (98), we arrive at

$$
i\left(A,\left(\Omega_{R} \backslash \bar{\Omega}_{r_{2}}\right) \cap P^{2}, P^{2}\right)=1 .
$$

Therefore $A$ has at least one fixed point on $\left(\Omega_{R} \backslash \bar{\Omega}_{r_{2}}\right) \cap P^{2}$. Hence (1) has at least one positive solution, which completes the proof.

Theorem 14. If (H1), (H2), (H4), (H7), and (H8) hold, then (1) has at least two positive solutions.

Proof. By (H8), we have

$$
\begin{aligned}
& \int_{0}^{1} f(s, \omega, \omega, \omega, \omega, \omega, \omega, \omega, \omega) \mathrm{d} s<\omega, \\
& \int_{0}^{1} g(s, \omega, \omega, \omega, \omega, \omega, \omega, \omega, \omega) \mathrm{d} s<\omega,
\end{aligned}
$$


for all $t \in[0,1]$ and all $(x, y) \in I_{\omega}^{8}$. Consequently, we have, for all $(u, v) \in \partial \Omega_{\omega} \cap P^{2}$,

$$
\begin{aligned}
& \left\|A_{1}(u, v)\right\|_{0}=A_{1}(u, v)(1) \\
& =\int_{0}^{1} s f\left(s,(B u)(s),(B u)^{\prime}(s), u(s), u^{\prime}(s),\right. \\
& \left.(B v)(s),(B v)^{\prime}(s), v(s), v^{\prime}(s)\right) \mathrm{d} s \\
& \leqslant \int_{0}^{1} f\left(s,(B u)(s),(B u)^{\prime}(s), u(s), u^{\prime}(s),\right. \\
& \left.(B v)(s),(B v)^{\prime}(s), v(s), v^{\prime}(s)\right) \mathrm{d} s \\
& \leqslant \int_{0}^{1} f(s, \omega, \omega, \omega, \omega, \omega, \omega, \omega, \omega) \mathrm{d} s \\
& <\omega=\|(u, v)\|, \\
& \left\|A_{2}(u, v)\right\|_{0}=A_{2}(u, v)(1) \\
& =\int_{0}^{1} s g\left(s,(B u)(s),(B u)^{\prime}(s), u(s), u^{\prime}(s),\right. \\
& \left.(B v)(s),(B v)^{\prime}(s), v(s), v^{\prime}(s)\right) \mathrm{d} s \\
& \leqslant \int_{0}^{1} g\left(s,(B u)(s),(B u)^{\prime}(s), u(s), u^{\prime}(s),\right. \\
& \left.(B v)(s),(B v)^{\prime}(s), v(s), v^{\prime}(s)\right) \mathrm{d} s \\
& \leqslant \int_{0}^{1} g(s, \omega, \omega, \omega, \omega, \omega, \omega, \omega, \omega) \mathrm{d} s \\
& <\omega=\|(u, v)\|, \\
& \left\|A_{1}(u, v)^{\prime}\right\|_{0}=A_{1}(u, v)^{\prime}(0) \\
& =\int_{0}^{1} f\left(s,(B u)(s),(B u)^{\prime}(s), u(s), u^{\prime}(s),\right. \\
& \left.(B v)(s),(B v)^{\prime}(s), v(s), v^{\prime}(s)\right) \mathrm{d} s \\
& \leqslant \int_{0}^{1} f(s, \omega, \omega, \omega, \omega, \omega, \omega, \omega, \omega) \mathrm{d} s \\
& <\omega=\|(u, v)\|, \\
& \left\|A_{2}(u, v)^{\prime}\right\|_{0}=A_{2}(u, v)^{\prime}(0) \\
& =\int_{0}^{1} g\left(s,(B u)(s),(B u)^{\prime}(s), u(s), u^{\prime}(s),\right. \\
& \left.(B v)(s),(B v)^{\prime}(s), v(s), v^{\prime}(s)\right) \mathrm{d} s \\
& \leqslant \int_{0}^{1} g(s, \omega, \omega, \omega, \omega, \omega, \omega, \omega, \omega) \mathrm{d} s \\
& <\omega=\|(u, v)\| .
\end{aligned}
$$

The preceding inequalities imply

$$
\|A(u, v)\|=\left\|\left(A_{1}(u, v), A_{2}(u, v)\right)\right\|<\omega=\|(u, v)\| ;
$$

thus

$$
(u, v) \neq \lambda A(u, v), \quad \forall(u, v) \in \partial \Omega_{\omega} \cap P^{2}, 0 \leqslant \lambda \leqslant 1 .
$$

Now Lemma 7 yields

$$
i\left(A, \Omega_{\omega} \cap P^{2}, P^{2}\right)=1 .
$$

By (H2), (H4), and (H7), we find that (51) and (98) hold. Note that we can choose $R>\omega>r_{2}$ in (51) and (98) (see the proofs of Theorems 11 and 13). Combining (51), (98), and (104), we obtain

$$
\begin{aligned}
& i\left(A,\left(\Omega_{R} \backslash \bar{\Omega}_{\omega}\right) \cap P^{2}, P^{2}\right)=0-1=-1, \\
& i\left(A,\left(\Omega_{\omega} \backslash \bar{\Omega}_{r_{2}}\right) \cap P^{2}, P^{2}\right)=1-0=1 .
\end{aligned}
$$

Therefore, $A$ has at least two fixed points, with one on $\left(\Omega_{R} \mid\right.$ $\left.\bar{\Omega}_{\omega}\right) \cap P^{2}$ and the other on $\left(\Omega_{\omega} \backslash \bar{\Omega}_{r_{2}}\right) \cap P^{2}$. Hence (1) has at least two positive solutions. This completes the proof.

\section{Examples}

Example 15. Let

$$
\begin{aligned}
& f(t, x, y):=\left(\sum_{i=1}^{4} a_{i} x_{i}+\sum_{i=1}^{4} a_{i+4} y_{i}\right)^{p}, \\
& g(t, x, y):=\left(\sum_{i=1}^{4} b_{i} x_{i}+\sum_{i=1}^{4} b_{i+4} y_{i}\right)^{q},
\end{aligned}
$$

where $a_{i}, b_{i}>0,1<p<2$, and $1<q<2$. Then (H1), (H2), (H4), and (H5) hold. By Theorem 11, (1) has at least one positive solution.

Example 16. Let

$$
\begin{aligned}
& f(t, x, y):=\left(\sum_{i=1}^{4} c_{i} x_{i}+\sum_{i=1}^{4} c_{i+4} y_{i}\right)^{p}, \\
& g(t, x, y):=\left(\sum_{i=1}^{4} d_{i} x_{i}+\sum_{i=1}^{4} d_{i+4} y_{i}\right)^{q},
\end{aligned}
$$

where $c_{i}, d_{i}>0,0<p<1$, and $0<q<1$. Then (H1), (H6), and (H7) hold. By Theorem 13, (1) has at least one positive solution. 
Example 17. Let

$$
\begin{aligned}
f(t, x, y):= & \lambda\left(\sum_{i=1}^{4} a_{i} x_{i}+\sum_{i=1}^{4} a_{i+4} y_{i}\right)^{p_{1}} \\
& +\lambda\left(\sum_{i=1}^{4} c_{i} x_{i}+\sum_{i=1}^{4} c_{i+4} y_{i}\right)^{p_{2}}, \\
g(t, x, y):= & \lambda\left(\sum_{i=1}^{4} b_{i} x_{i}+\sum_{i=1}^{4} b_{i+4} y_{i}\right)^{q_{1}} \\
& +\lambda\left(\sum_{i=1}^{4} d_{i} x_{i}+\sum_{i=1}^{4} d_{i+4} y_{i}\right)^{q_{2}}
\end{aligned}
$$

where $a_{i}, b_{i}, c_{i}, d_{i}>0,0<p_{1}<1, p_{2}>1,0<q_{1}<1$, and $q_{2}>$ $1 ; \lambda>0$ is sufficiently small. Then (H1), (H2), (H4), (H7), and (H8) hold. By Theorem 14, (1) has at least two positive solutions.

\section{Conflict of Interests}

The authors declare that there is no conflict of interests for any of them.

\section{References}

[1] T. M. Atanackovic, Stability Theory of Elastic Rods. Series on Stability, Vibration and Control of Systems, Series A, World Scientific, Singapore, 1997.

[2] C. P. Gupta, "Existence and uniqueness theorems for the bending of an elastic beam equation," Journal of Applied Analysis, vol. 26, pp. 289-304, 1988.

[3] Y. Li, Y. Guo, and G. Li, "Existence of positive solutions for systems of nonlinear third-order differential equations," Communications in Nonlinear Science and Numerical Simulation, vol. 14, no. 11, pp. 3792-3797, 2009.

[4] H. Lü, H. Yu, and Y. Liu, "Positive solutions for singular boundary value problems of a coupled system of differential equations," Journal of Mathematical Analysis and Applications, vol. 302, no. 1, pp. 14-29, 2005.

[5] H. Su, Z. Wei, X. Zhang, and J. Liu, "Positive solutions of n-order and m-order multi-point singular boundary value system," Applied Mathematics and Computation, vol. 188, no. 2, pp. 12341243, 2007.

[6] Z. Yang, "Existence of positive solutions for a system of generalized Lidstone problems," Computers and Mathematics with Applications, vol. 60, no. 3, pp. 501-510, 2010.

[7] Z. Cao and D. Jiang, "Periodic solutions of second order singular coupled systems," Nonlinear Analysis: Theory, Methods \& Applications, vol. 71, no. 9, pp. 3661-3667, 2009.

[8] J. Chu, D. O’Regan, and M. Zhang, "Positive solutions and eigenvalue intervals for nonlinear systems," Proceedings of the Indian Academy of Sciences: Mathematical Sciences, vol. 117, no. 1, pp. 85-95, 2007.

[9] D. R. Dunninger and H. Wang, "Multiplicity of positive radial solutions for an elliptic system on an annulus," Nonlinear Analysis: Theory, Methods \& Applications, vol. 42, no. 5, pp. 803811,2000 .
[10] X. Cheng, "Existence of positive solutions for a class of secondorder ordinary differential systems," Nonlinear Analysis: Theory, Methods \& Applications, vol. 69, pp. 3042-3049, 2008.

[11] K. Wang and Z. Yang, "Positive solutions for a system of second-order boundary value problems involving first order derivatives," Electronic Journal of Differential Equations, vol. 2012, no. 52, pp. 1-15, 2012.

[12] Z. Yang and J. Sun, "Positive solutions of a fourth-order boundary value problem involving derivatives of all orders," Communications on Pure and Applied Analysis, vol. 11, no. 5, pp. 1615-1628, 2012.

[13] D. Guo and V. Lakshmikantham, Nonlinear Problems in Abstract Cones, Academic Press, Boston, Mass, USA, 1988.

[14] Z. Yang, "Positive solutions for a system of nonlinear Hammerstein integral equations and applications," Applied Mathematics and Computation, vol. 218, pp. 11138-11150, 2012.

[15] A. Berman and J. Plemmons, Nonnegative Matrices in the Mathmatical Sciences, Academic Press, New York, NY, USA, 1979.

[16] Z. Yang and L. Kong, "Positive solutions of a system of second order boundary value problems involving first order derivatives via and $\mathbb{R}_{+}^{n}$-monotone matrices," Nonlinear Analysis: Theory, Methods \& Applications, vol. 75, no. 4, pp. 2037-2046, 2012. 


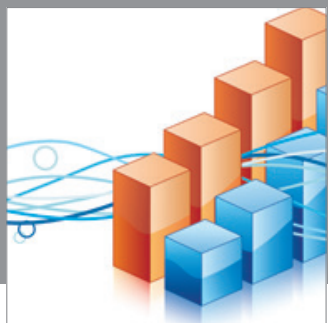

Advances in

Operations Research

mansans

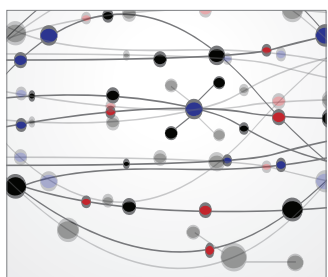

The Scientific World Journal
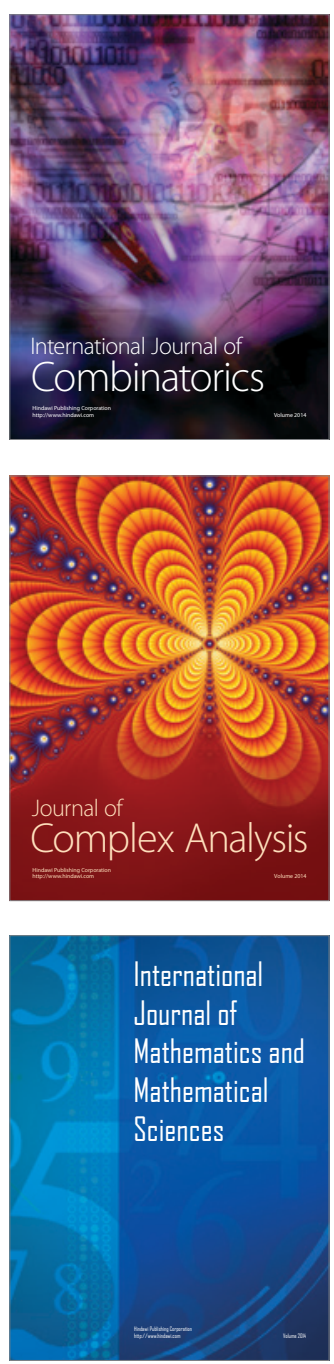
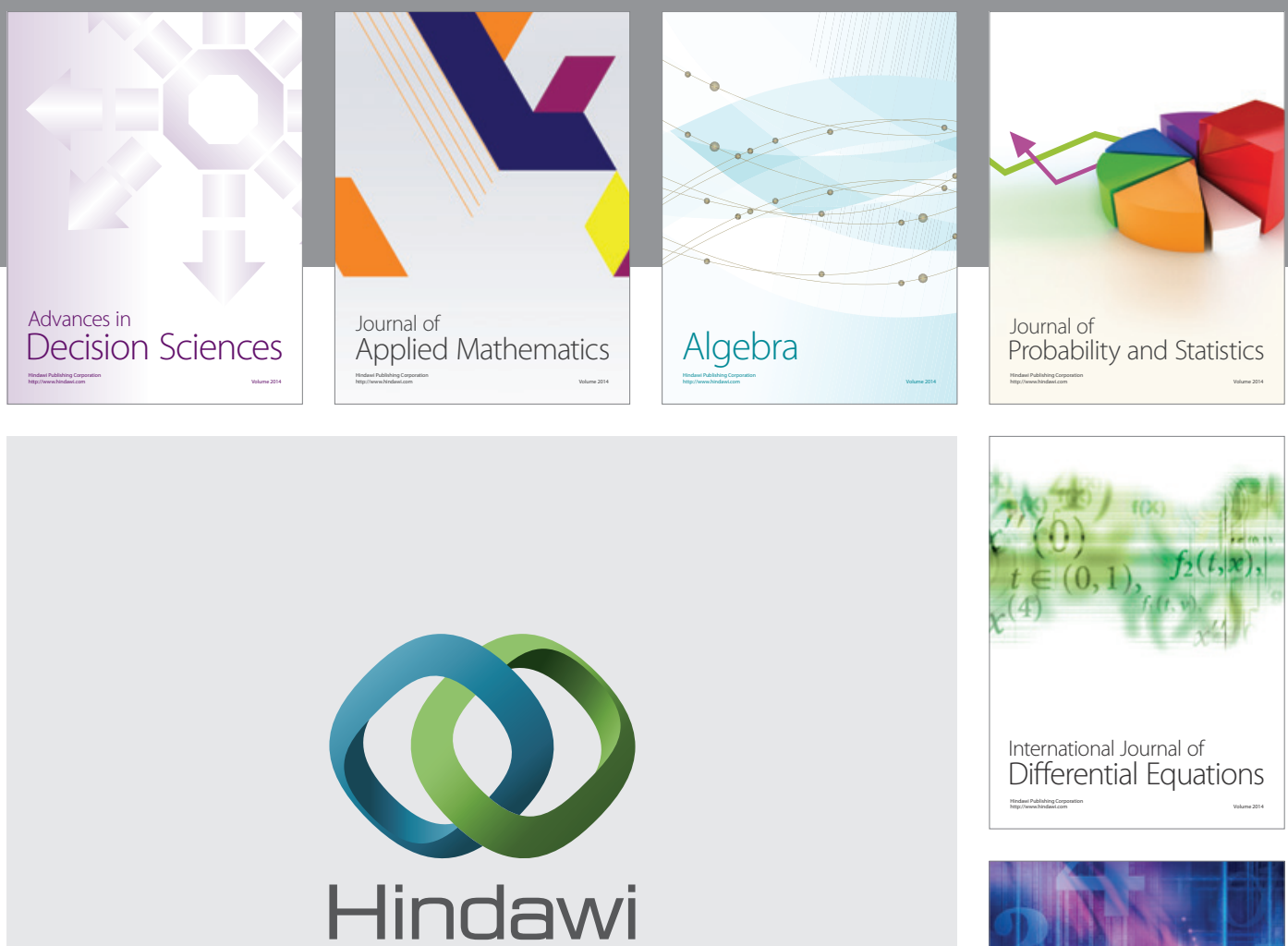

Submit your manuscripts at http://www.hindawi.com
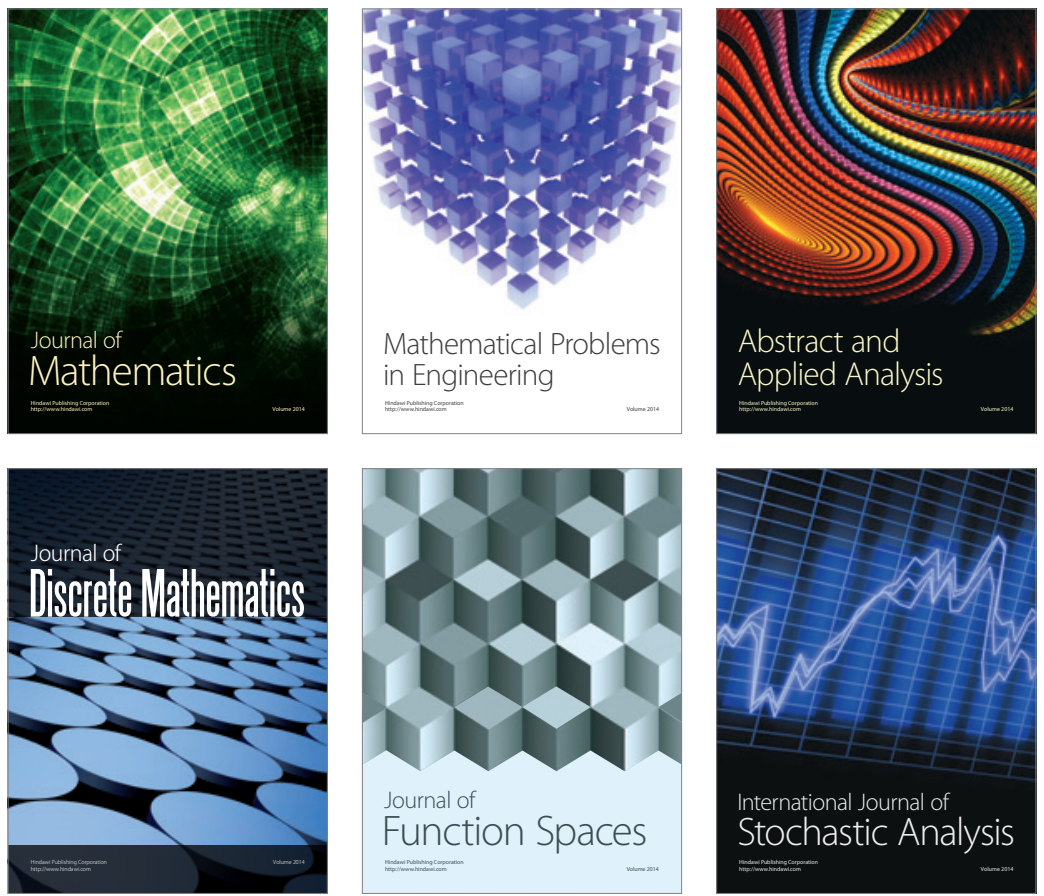

Journal of

Function Spaces

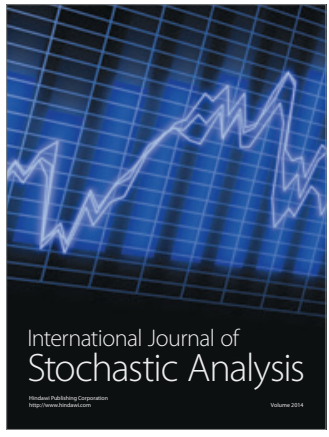

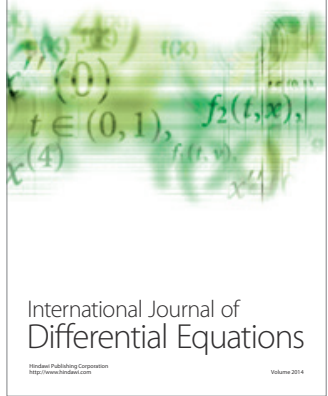
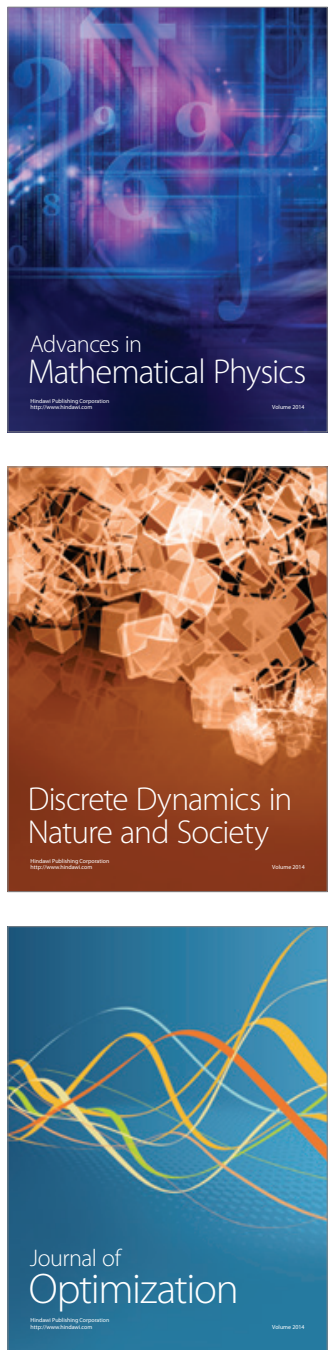